\title{
Neffropatia diabética em cães: revisão de literatura
}

\section{Diabetic nephropathy in dogs: literature review}

\section{Resumo}

O diabetes mellitus que se desenvolve por deficiência de insulina e hiperglicemia crônica usualmente apresenta complicações que incluem catarata, retinopatia, infecções recorrentes e cetoacidose. A hiperglicemia crônica pode promover complicações a longo prazo como a nefropatia diabética. Nos humanos, a nefropatia diabética é caracterizada principalmente por lesão glomerular e geralmente está associada a hipertensão arterial sistêmica. Dentre os mecanismos propostos para a fisiopatologia da nefropatia diabética tem sido referida a lesão glomerular, assim como a lesão nos túbulos renais. Contudo, até o momento existem poucos estudos investigando a relação entre diabetes mellitus e lesão renal em cães. Esta revisão tem por objetivos esclarecer os possíveis mecanismos fisiopatológicos da nefropatia diabética e discutir o conhecimento disponível sobre sua ocorrência em cães.

\section{Abstract}

Diabetes mellitus caused by insulin deficiency and chronic hyperglycemia usually presents complications including cataract, retinopathy, recurrent infections and ketoacidosis. Chronic hyperglycemia can cause long-term complications such as diabetic nephropathy. In humans, diabetic nephropathy is characterized mainly by glomerular injury and usually it is associated with systemic hypertension. The mechanisms proposed for the pathogenesis of diabetic nephropathy could be glomerular injury, as well as renal tubular damage. However, there are few studies analyzing the relationship between diabetes mellitus and kidney damage in dogs. The objective of this review is to clarify the pathophysiological mechanisms of diabetic nephropathy and to analyze the available information about its occurrence in dogs. 
Cínthia Ribas Martorelli ${ }^{1}$

Márcia Mery Kogika ${ }^{1}$

Douglas Segalla Caragelasco
Av. Prof. Dr. Orlando Marques Paiva, 87

Cidade Universitária, São Paulo/SP

CEP: 05508-270

$\triangle$ cinthiarm@usp.br

\section{Palavras-chave}

Canino. Rim. Hiperglicemia.

Diabetes mellitus.

\section{Keywords}

Canine. Kidney. Hyperglycemia.

Diabetes mellitus.

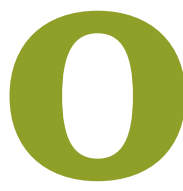

diabetes mellitus insulinodependente é a endocrinopatia mais comum entre cães, e se define como um estado hiperglicêmico crônico causado pela deficiência na síntese ou pelo comprometimento na ação da insulina (deficiência relativa ou absoluta), ou ainda por fatores antagonistas da insulina (NELSON, 2015). Complicações a curto prazo decorrentes do estado diabético são comuns em cães, sendo as mais frequentes a cegueira devido ao desenvolvimento de catarata, a pancreatite crônica e as infecções recidivantes do trato urinário, do sistema respiratório e da pele (HESS et al., 2000).

Nos humanos, as complicações crônicas mais graves, como nefropatia, retinopatia e neuropatia, são observadas após 10 a 20 anos do diagnóstico do diabetes mellitus, e mesmo que em cães estas pareçam ser raras, ainda não foram realizadas investigações de longa duração em caninos (WARRAM et al., 1996).

As complicações crônicas do diabetes mellitus ocorrem nos tecidos que não dependem de insulina para entrada de glicose no interior das células - a retina, os rins e os nervos periféricos são os tecidos-alvos (ROSSING; HOUGAARD; PARVING, 2002; OH et al., 2012). Baseando-se nos estudos realizados em humanos diabéticos, foram propostas duas hipóteses para explicar o desenvolvimento das complicações tardias do diabetes mellitus nesses tecidos. A primeira se refere à presença de anormalidades metabólicas, secundárias ao estado hiperglicêmico, resultando em alterações estruturais e funcionais de células em que o excesso de glicose no meio intracelular afeta o metabolismo lipídico e aumenta a produção de radicais livres, 
provavelmente envolvidos na patogenia das complicações do diabetes mellitus. A segunda se refere à existência de uma vasculopatia como causa principal, provocando alterações estruturais e funcionais nos vasos sanguíneos. A nefropatia, a neuropatia e a retinopatia são complicações microvasculares do diabetes mellitus (WARRAM et al., 1996; CARAMORI; PARKS; MAUER, 2013).

Nos pacientes humanos, a nefropatia diabética é uma complicação bastante frequente, reconhecida como importante fator no desenvolvimento de doença renal crônica. Nos estágios iniciais, caracteriza-se por hiperfiltração e hiperperfusão glomerular, associadas ao desenvolvimento de alterações morfológicas como espessamento da membrana basal glomerular e expansão tubulointersticial, porém sem manifestação clínica. As alterações morfológicas iniciais observadas na nefropatia diabética incluem hipertrofia glomerular, espessamento da membrana basal e tubular, além de expansão da matriz extracelular do mesângio glomerular (ROSSING; HOUGAARD; PARVING, 2002; NAUTA et al., 2011; Moriya et al., 2012; CARAMORI; PARKS; MAUER, 2013).

Em seres humanos, o aumento da excreção urinária de albumina indica a evolução da patologia, que passa a ser denominada nefropatia diabética incipiente. Nos estágios mais avançados foram observadas outras alterações, como glomeruloesclerose, falência dos capilares glomerulares, degeneração e fibrose tubulointersticial, associadas ao declínio da função renal (ROSSING; HOUGAARD; PARVING, 2002; NAUTA et al., 2011; NIELSEN et al., 2011; Moriya et al., 2012; Khazim et al., 2013).

Assim, a alteração característica da nefropatia diabética é a proteinúria, um marcador de gravidade e de progressão para doença renal crônica nesses pacientes. Ainda, a patogenia da nefropatia diabética se relaciona com a duração do diabetes mellitus, o controle glicêmico e com fatores genéticos (STOLAR, 2010).

Nos seres humanos com nefropatia diabética, foi demonstrado que a glomerulopatia ocorre antes das alterações túbulo-intersticiais, o que não foi observado em ratos diabéticos (KANG et al., 2005; Nadarajah et al., 2012; Pourghasem; Nasiri; SHAFI, 2014). Assim, os ratos com diabetes mellitus experimental, induzida por aloxano ou por estreptozotocina, podem não ser o modelo ideal para o estudo da nefropatia diabética humana, pois apresentam principalmente glomeruloesclerose e lesões vasculares (KANG et al., 2005; ALPERS; HUDKINS, 2011; NIELSEN et al., 2011; Pourghasem; Nasiri; SHAFI, 2014). O depósito de glicogênio nas células tubulares também foi observado nos humanos com nefropatia diabética, principalmente nos ductos coletores e na alça de Henle descendente, que provavelmente ocorre no estágio terminal da nefropatia diabética (KANG et al., 2005; Pourghasem; Nasiri; SHAFI, 2014).

Em humanos diabéticos, as lesões tubulares foram observadas na biópsia, porém ainda não está totalmente elucidado se a proteinúria é inicialmente resultante de alterações na barreira de filtração glomerular, da perda da capacidade de reabsorção do túbulo proximal ou de lesão intersticial. Ainda, é provável que a lesão das células tubulares aumente da excreção urinária de proteínas por comprometer a reabsorção dessas normalmente filtradas pelo glomérulo - o desenvolvimento de doença glomerular acarreta lesão tubulointersticial exacerbada e redução ainda maior de reabsorção. Talvez a "piora da proteinúria glomerular" possa refletir maior prejuízo na reabsorção do túbulo proximal frente às proteínas filtradas pelo glomérulo, logo a magnitude da proteinúria pode decorrer do grau de lesão tubular e/ou glomerular e/ou intersticial verificada (NAUTA et al., 2011; NIELSEN et al., 2011; KIM et al., 2013).

Ainda nos humanos diabéticos, acredita-se existir lesão em diferentes locais dos néfrons, como nas células endoteliais glomerulares, na membrana basal glomerular, nos podócitos e no túbulo proximal (JEFFERSON; SHANKLAND; PICHLER, 2008). Dessa forma, os mecanismos propostos incluem: hiperglicemia crônica; lesão nos podócitos; lesão tubulointersticial; ativação do sistema-renina-angiotensina-aldosterona; ação de fatores de crescimento pró-fibróticos e subsequente fibrose intesticial; ação de citocinas inflamatórias e lesões vasculares.

A falta de um controle glicêmico adequado em humanos promove inicialmente hipertrofia e espessamento da membrana basal glomerular, aumento na síntese proteica da matriz mesangial, além de maior produção de prostaglandinas vasodilatadoras, contribuindo para hiperperfusão, hipertensão e hiperfiltração intraglomerular (LOPES-VIRELLA et al., 1979). Paralelamente, em estudo experimental executado com ratos diabéticos submetidos a um controle glicêmico inadequado, foi observado o aumento da taxa de filtração glomerular, ocasionado por hipertensão intraglomerular e maior reabsorção de sódio e glicose (JENSEN et al., 1987), achados semelhantes aos observados em pacientes humanos (MOGENSEN, 1971). Rasch (1979) ressalta que o controle glicêmico inadequado em ratos diabéticos se associa a uma maior excreção urinária de albumina. Lee et al. (1974) ressaltam que essas lesões podem ser minimizadas ou evitadas com controle glicêmico adequado, e que dependem da duração e da intensidade do tratamento com insulina.

Lee et al. (1974) observaram que as lesões renais, características do diabetes mellitus, são revertidas quando o rim é transplantado para um animal não diabético. Assim, 
a hiperglicemia ativa estimula a atuação de outros mecanismos envolvidos no desenvolvimento da nefropatia diabética, tais como: maior produção de matriz extracelular do mesângio glomerular; alterações bioquímicas da matriz extracelular; alteração de fatores hemodinâmicos que promovem hipertrofia renal (FARIA, 2001).

A hiperglicemia em humanos também altera a hemodinâmica renal, com posterior aumento da taxa de filtração glomerular e do fluxo plasmático renal logo nos estágios iniciais da nefropatia diabética, também contribuindo para o aparecimento de proteinúria e alterações histológicas nos rins (VORA; ANDERSON; BRENNER, 1994). Em modelos animais este fato também foi observado, resultando no aumento da pressão intraglomerular com consequente lesão endotelial e da produção de matriz extracelular, fatores envolvidos na progressão na nefropatia diabética, mesmo sob controle glicêmico adequado (ZATZ et al., 1985, 1986).

A hipertrofia renal se associa à hipertrofia glomerular e ao aumento da taxa de filtração glomerular no estágio inicial do diabetes mellitus, e também à atuação de fatores de crescimento, tanto em ratos quanto em humanos diabéticos. Porém, o mecanismo e o papel exato destes fatores ainda não estão totalmente elucidados no desenvolvimento da nefropatia diabética (DOI et al., 1988; BACH et al., 2000; FLYVBJERG, 2000; FARIA, 2001).

Outro mecanismo bastante discutido é a ocorrência de lesão nos podócitos em humanos que, juntamente com a hiperglicemia, atuam como principais responsáveis por lesão nas células endoteliais glomerulares. Paralelamente há queda do número de podócitos (também denominado de podocitopenia), permitindo a passagem de proteínas para a urina (proteinúria), podendo evoluir para glomeruloesclerose e comprometimento da função renal (FIORETTO et al., 1992; SUSZTAK et al., 2006).

O mecanismo de podocitopenia não está totalmente elucidado, mas acredita-se que a hiperglicemia promova a liberação de radicais livres, causando a apoptose destas células (FIORETTO et al., 1992; SUSZTAK et al., 2006). Geralmente, o número de podócitos se preserva nos estágios iniciais da nefropatia diabética, porém alguns fatores como descolamento dos podócitos do glomérulo, apoptose de podócitos, que coincide com maior excreção urinária de albumina, incapacidade para proliferação dos podócitos, alteração do citoesqueleto e perda da carga negativa podem desencadear a podocitopenia. Posteriormente, ocorre diminuição da capacidade reabsortiva tubular, com progressiva fibrose intersticial (MAUER, 1994; SUSZTAK et al., 2006).

A patogenia da nefropatia diabética em seres humanos também é marcada pela ativação do sistema renina-angiotensina-aldosterona. Dessa forma, a renina produzida converte o angiotensinogênio em angiotensina I, e a enzima conversora de angiotensina transmuta a angiotensina I em angiotensina II, a qual atua localmente em diferentes tecidos, como rins, coração e vasos sanguíneos, de maneira sistêmica ao promover a homeostase cardiovascular por meio de receptores específicos. Nos rins, a angiotensina II estimula a retenção hídrica no túbulo proximal ao estimular a produção de aldosterona pela zona glomerular das glândulas adrenais. Esta proteína está envolvida na patogenia da nefropatia diabética, pois altera a matriz extracelular glomerular com consequente hipertrofia, associada à deposição de colágeno na matriz extracelular, que é característica deste quadro clínico (FERREIRA; ZANELLA, 2000). Em associação a este quadro, a ativação do sistema renina-angiotensina-aldosterona causa constrição seletiva da arteríola eferente, aumentando também a pressão intraglomerular, contribuindo assim no desenvolvimento e perpetuação da nefropatia diabética (MYERS et al., 1982).

Nos pacientes humanos as alterações vasculares também estão presentes na nefropatia diabética e são representadas pela arteriosclerose e pela hialinose das arteríolas glomerulares aferente e eferente. Também foram observadas lesões exsudativas no espaço subendotelial e sobre a camada parietal da cápsula de Bowman's. Estas lesões por sua vez resultam em adesão do tufo glomerular seguidas da ruptura da membrana basal glomerular, retração e remodelamento glomerular. Ainda foram verificados microaneurismas nas alças dos capilares glomerulares e posteriormente constatou-se lesão tubulointersticial (MAUER, 1994).

Nos cães, as complicações crônicas mais graves do diabetes mellitus como nefropatia diabética parecem ser raras, pois em humanos esse tipo de complicação pode necessitar de um tempo longo de evolução do diabetes mellitus, situando-se em torno de dez a vinte anos (HESS et al., 2000; BLOOM; RAND, 2013; HERRING; PANCIERA; WERRE, 2014).

Em ensaio experimental realizado em cães da raça Beagle, os animais foram divididos em dois grupos e submetidos a nefrectomia unilateral para a investigação da nefropatia diabética. O grupo 1 foi composto por cães que tinham controle glicêmico regular com o único intuito de evitar cetoacidose diabética, e o grupo 2, por animais que apresentaram controle glicêmico adequado pela administração de insulina duas vezes ao dia. Dos animais dos dois grupos experimentais foram coletados fragmentos de tecido renal, antes e após 6 e 12 meses da indução do diabetes mellitus, submetidos a análise por microscopia óptica e eletrônica (STEFFES et al., 1982). Após um ano de evolução do diabetes mellitus, todos os 
cães do grupo 1 apresentaram no exame histopatológico dos rins as alterações: expansão mesangial glomerular, glomerulonefropatia membranosa associada com fusão dos podócitos, espessamento da membrana basal glomerular e tubular mesangial, aumento da produção de matriz extracelular do mesângio glomerular, presença de depósitos subendoteliais, fibrose glomerular e glomeruloesclerose (nodular ou não), demonstrando que o cão pode ser um modelo para o estudo da nefropatia diabética, e que o estabelecimento de controle glicêmico adequado pode prevenir o aparecimento de nefropatia diabética (STEFFES et al., 1982).

Kern e Ergerman (1990), em investigação experimental realizada com cães, constataram que o controle glicêmico inadequado relacionava-se com maior excreção urinária de proteínas, e que poderia ser, em parte, resultante de lesão glomerular. Ainda observaram que o grau de excreção de proteína urinária variou com o controle glicêmico, e no exame histopatológico dos rins foram constatadas obliteração glomerular e expansão mesangial.

A fisiopatologia do aumento da excreção urinária de proteínas no diabetes mellitus em cães é desconhecida, porém sabe-se que diferentes mecanismos como anormalidades bioquímicas, morfológicas e hemodinâmicas estão envolvidos. A primeira hipótese seria a presença de lesão glomerular e, posteriormente, a excessiva secreção tubular de proteínas como a beta-microglobulina. Assim, nos estudos de indução experimental de diabetes mellitus do tipo 1 nos cães foi observado o desenvolvimento de doença renal à semelhança dos pacientes humanos (STEFFES et al., 1982; KERN; ERGERMAN, 1990; BLOOM; RAND, 2013; HERRING; PANCIERA; WERRE, 2014).

Struble et al. (1998) realizaram uma avaliação pontual com cães diabéticos em que foi observada proteinúria em $20 \%$ dos casos avaliados por meio do aumento da razão proteína: creatinina urinária (RPC) maior que 0,5 e da razão albumina: creatinina urinária (RAC) maior que 0,03 . Constataram que a proteinúria foi associada com hipertensão sistêmica ( $24 \%$ dos cães avaliados) e com a duração do diabetes mellitus, entretanto a importância clínica desses achados ainda não foi totalmente esclarecida.

Kogika et al. (2007), em uma avaliação clínica e de observação pontual em cães diabéticos, investigaram a ocorrência de microalbuminúria e de hipertensão arterial sistêmica e verificaram a relação das alterações com o tempo de evolução do diabetes mellitus. Para isso foi empregado um teste semiquantitativo (Early Renal Damage - HealthScreen Test - Heska Corporation, Loveland, CO), com resultado negativo para 52,9\% dos casos, "fraco positivo" em $6 \%$, "médio positivo" em $23,5 \%$ e "alto positivo" em 17,6\% dos casos. Nos cães com resultados médio e alto positivo, a duração média do diabetes mellitus foi de 18,2 meses, sugerindo que o tempo de evolução da doença poderia estar relacionado com a proteinúria, e não com a hipertensão arterial sistêmica.

Contudo, a hipertensão arterial sistêmica em cães diabéticos ainda requer estudos verticalizados e de acompanhamento sequencial, pois há escassez de resultados concretos que justifiquem a semelhança na evolução do quadro observado em cães com o registrado na medicina humana, em que a hipertensão arterial sistêmica se correlaciona à duração do diabetes mellitus e ao controle glicêmico dos pacientes.

$\mathrm{Na}$ avaliação pontual em cães com diabetes mellitus, Kogika et al. (2007) constataram que $46 \%$ dos animais apresentaram hipertensão arterial sistêmica, positivamente correlacionada com a duração do diabetes mellitus. Entretanto, para que uma avaliação adequada da pressão arterial sistêmica seja realizada, a mensuração deverá ser tomada em diversos momentos, ou seja, é preciso confirmar se os valores aumentados da pressão arterial são mantidos ou sustentados; o efeito da hipertensão decorrente de estresse deverá ser descartado, e além disso são necessários alguns cuidados durante o procedimento de mensuração, tais como a ambientalização do animal para amenizar o estresse, bem como a determinação da largura correta do manguito - que deve ser selecionada a partir da mensuração do diâmetro do membro (preferencialmente torácico) em centímetros -, a qual deve corresponder a $40 \%$ do comprimento da circunferência do membro (BROWN et al., 2007). Destaca-se ainda que o animal deverá ser mantido em uma única posição durante todo o procedimento (por exemplo, em decúbito esternal), com o esfigmomanômetro sempre na altura do átrio direito (BROWN et al., 2007). O registro correto será obtido pela determinação da média de cinco a sete aferições e ainda, após uma semana, deverá ser confirmado se a pressão sistólica é superior a $150 \mathrm{mmHg}$ (que denota presença de hipertensão arterial sistêmica), desde que não haja evidências de lesões em órgãos-alvo relacionadas à hipertensão, com cuidado especial para as lesões oculares retinianas (BROWN et al., 2007).

Os mecanismos etiológicos exatos da nefropatia diabética no cão ainda não são conhecidos, mas provavelmente o processo conta com uma etiologia multifatorial e, no presente momento, os textos em medicina veterinária têm extrapolado as informações disponíveis na medicina humana (BLOOM; RAND, 2013; HERRING; PANCIERA; WERRE, 2014).

Em Medicina Veterinária, a avaliação da condição da proteinúria ser de origem renal e patológica foi estabelecida por Lees et al. (2005), que destacam a necessidade da 
confirmação da sua persistência por um período mínimo de quinze e máximo de trinta dias. Ressalta-se, contudo, que essa persistência deverá ser avaliada após a exclusão de diversas outras doenças concomitantes como: infecção do trato urinário, neoplasias, urolitíase, ureterolitíase, nefrolitíase, hiperadrenocorticismo, doenças infecciosas, doenças inflamatórias e uso de alguns medicamentos, como glicocorticóides e inibidores da enzima conversora de angiotensina.

Nas investigações realizadas por Struble et al. (1998), Mazzi et al. (2008) e Herring, Panciera e Werre (2014) não foram adotados os períodos propostos por Lees et al. (2005), portanto os resultados de tais ensaios não permitem a afirmação de que a proteinúria foi decorrente de lesão renal, consequentemente patológica.

Mazzi et al. (2008) constataram albuminúria e hipertensão sistêmica, respectivamente, em $25 \%$ e $35 \%$ dos cães diabéticos examinados. No entanto, os cães foram avaliados apenas uma única vez e, de acordo com os critérios estabelecidos por Lees et al. (2005), não se confirma portanto a existência de proteinúria renal patológica. Já Herring, Panciera e Werre (2014) constataram a ocorrência de albuminúria em $55 \%$ dos casos de cães diabéticos que acompanharam.

Martorelli et al. (2016), em uma observação sequencial realizada com cães diabéticos, não verificaram o desenvolvimento de nefropatia diabética, investigada por meio da constatação de proteinúria renal patológica, durante o período do estudo, o que provavelmente não ocorreu devido ao bom controle glicêmico adotado, como também ao menor tempo de acompanhamento dos animais avaliados.

Nos cães diabéticos, a nefropatia diabética talvez apresente um curso diferente do observado em humanos, pois a hipertensão arterial sistêmica não é uma alteração frequente em cães com diabetes mellitus. Deste modo, torna-se necessária a realização de investigações longitudinais que avaliem sequencialmente a proteinúria e identifiquem as proteínas específicas decorrentes de lesões glomerulares ou tubulares para que seja determinada a sua persistência e confirmada a proteinúria renal patológica. Essas avaliações deverão ser realizadas juntamente com a mensuração da pressão arterial sistêmica. Também deve ser considerado que o desenvolvimento da nefropatia diabética pode ser prevenido em cães submetidos a um controle glicêmico adequado (MARTORELLI et al., 2016). Estudos de longo prazo efetuados em cães com diabetes mellitus não tratado adequadamente poderiam confirmar a hipótese de que a falta de controle glicêmico adequado causaria lesões renais, no entanto, certamente tal estudo seria considerado não plausível e antiético.

\section{Considerações finais}

Em Medicina Veterinária ainda há poucas investigações de longa duração avaliando a presença de lesão renal com proteinúria em cães diabéticos. Ainda falta confirmação definitiva da existência de proteinúria renal patológica, persistente e não associada a outras doenças concomitantes que também poderiam causar a mesma alteração. Portanto, ainda não existem evidências suficientes para atestar que cães com diabetes mellitus apresentem risco de nefropatia diabética, como ocorre em seres humanos.

\section{Agradecimentos}

Agradecimento à Coordenação de Aperfeiçoamento de Pessoal de Nível Superior (CAPES). *

\section{Referências}

ALPERS, C. E.; HUDKINS, M. S. Mouse models of diabetic nephropathy. Current Opinion in Nephrology and Hypertension, Philadelphia, v. 20, n. 3, p. 278-284, 2011.

$\mathrm{BACH}, \mathrm{L}$. A. et al. Aminoguanidine ameliorates changes in the IGF system in experimental diabetic nephropath. Nephrology Dialysis Transplantation, Oxford, v. 15, n. 3, p. 347-354, 2000.

BLOOM, C. A.; RAND, J. S. Diabetes and the kidney in human and veterinary medicine. Veterinary Clinics of North America: Small Animal Practice, Philadelphia, v. 43, n. 2, p. 351-365, 2013.

BROWN, S. et al. Guidelines for the identification, evaluation, and management of systemic hypertension in dogs and cats. Journal of Veterinary Internal Medicine, Malden, v. 21, n. 3, p. 542-558, 2007.

CARAMORI, M. L.; PARKS, A.; MAUER, M. Renal lesions predict progression of diabetic nephropathy in type 1 diabetes. Journal of the American Society of Nephrology, Washington, v. 24, n. 7 , p. 1175-1181, 2013.

DOI, T. et al. Progressive glomerulosclerosis develops in transgenic mice chronically expressing growth hormone and growth releasing factor but not in those expressing insulin-like growth factor. American Journal Pathology, Philadelphia, v. 131, n. 3, p. 398403, 1988.

FARIA, J. B. L. Atualização em fisiologia e fisiopatologia: patogênese da nefropatia diabética. Jornal Brasileiro de Nefrologia, São Paulo, v. 23 , n. 2 , p. $121-129,2001$.

FERREIRA, S. R. G.; ZANELLA, M. T. Sistema renina-angiotensinaaldosterona e nefropatia diabética. Revista Brasileira de Hipertensão, Rio de Janeiro, v. 7, n. 3, p. 268-274, 2000.

FIORETTO, P. et al. An overview of renal pathology in insulindependent diabetes mellitus in relationship to altered glomerular hemodynamics. American Journal Kidney Disease, Philadelphia, v. 20 , n. 6 , p. 549-558, 1992. 
FLYVBJERG, A. Putative pathophysiological role of growth factors and cytokines in experimental diabetic kidney disease. Diabetologia, Berlin, v. 43, n. 10, p. 1205-1223, 2000.

HERRING, I. P.; PANCIERA, D. L.; WERRE, S. R. Longitudinal prevalence of hypertension, proteinuria, and retinopathy in dogs with spontaneous diabetes mellitus. Journal of Veterinary Internal Medicine, Malden, v. 28, n. 2, p. 488-495, 2014.

HESS, R. S. et al. Concurrent disorders in dogs with diabetes mellitus: 221 cases (1993-1998). Journal of American Veterinary Medical Association, Ithaca, v. 217, n. 8, p. 11661173, 2000.

JEFFERSON, J. A.; SHANKLAND, S. J.; PICHLER, R. H. Proteinuria in diabetic kidney disease: a mechanistic viewpoint. Kidney International, New York, v. 74, n. 1, p. 22-36, 2008.

JENSEN, P. K. et al. Strict metabolic control and renal function in the streptozotocin diabetic rat. Kidney International, New York, v. 31, n. 1, p. $47-51,1987$

KANG, J. et al. Glycogen accumulation in renal tubules, a key morphological change in the diabetic rat kidney. Acta Diabetologica, Berlin, v. 42, n. 2, p. 110-116, 2005.

KERN, T. S.; ERGERMAN, R. L. Arrest of glomerulopathy in diabetic dogs by improved glycaemic control. Diabetologia, Berlin, v. 33, n. 9, p. 522-525, 1990.

Khazim, K. et al. The antioxidant silybin prevents high glucoseinduced oxidative stress and podocyte injury in vitro and in vivo. American Journal of Physiology: Renal Physiology, Bethesda, v. 305, n. 5, p. F691-F700, 2013.

KIM, S. S. et al. Urinary cystatin $C$ and tubular proteinuria predict progression of diabetic nephropathy. Diabetes Care, Alexandria, v. 36, n. 3, p. 656-661, 2013

KOGIKA, M. M. et al. Microalbuminuria in dogs with diabetes mellitus. Journal of Veterinary Internal Medicine, Lakewood, v. 21, n. 3, p. $647,2007$.

LEE, C. S. et al. Renal transplantation in diabetes mellitus in the rat. The Journal of Experimental Medicine, New York, v. 139, n. 4 , p. $793-800,1974$

LEES, G. E. et al. Assessment and management of proteinuria in dogs and cats: 2004 ACVIM Forum Consensus Statement (small animal). Journal of Veterinary Internal Medicine, Lakewood, v. 19, n. 3, p. 377-385, 2005.

LOPES-VIRELLA, M. F. et al. Early diagnosis of renal malfunction in diabetes. Diabetologia, Berlin, v. 16, n. 3, p. 165-171, 1979.

MARTORELLI, C. R. et al. Sequential evaluation of proteinuria, albuminuria and urinary protein electrophoresis in dogs with diabetes mellitus. Online Journal of Veterinary Research, Queensland, v. 20, n. 8, p. 547-556, 2016.
MAUER, S. M. Structural-functional correlations of diabetic nephropathy. Kidney International, New York, v. 45, n. 2, p. 612-622, 1994.

MAZZI, A. et al. Ratio of urinary protein to creatinine and albumin to creatinine in dogs with diabetes mellitus and hyperadrenocorticism. Veterinary Research Communication, v. 32, p. 299-301, 2008. Supplement 1.

MOGENSEN, C. E. Glomerular filtration rate and renal plasma flow in short-term and long-term juvenile diabetes mellitus. Scandinavian Journal of Clinical and Laboratory Investigation, Oslo, v. 28, n. 1, p. 91-100, 1971.

Moriya, T. et al. Glomerular hyperfiltration and increased glomerular filtration surface are associated with renal function decline in normo and microalbuminuric type 2 diabetes. Kidney International, New York, v. 81, n. 5, p. 486-493, 2012.

MYERS, B. D. et al. Mechanisms of proteinuria in diabetic nephropathy: a study of glomerular barrier function. Kidney International, New York, v. 21, n. 4, p. 633-641, 1982.

Nadarajah, R. et al. Podocyte-specific overexpression of human angiotensin-converting enzyme 2 attenuates diabetic nephropathy in mice. Kidney International, New York, v. 82, n. 3, p. 292-303, 2012.

NAUTA, F. L. et al. Glomerular and tubular damage markers are elevated in patients with diabetes. Diabetes Care, Alexandria, v. 34, n. 4 , p. $975-981,2011$

NELSON, R. W. Canine diabetes mellitus. In: FELDMAN, E. C. et al. Canine and feline endocrinology. 4. ed. St. Louis: Elsevier, 2015. p. 213-257.

NIELSEN, S. E. et al. Tubular markers do not predict the decline in glomerular filtration rate in type 1 diabetic patients with overt nephropathy. Kidney International, New York, v. 79, n. 10 , p. $1113-1118,2011$

$\mathrm{OH}, \mathrm{S}$. W. et al. Clinical implications of pathologic diagnosis and classification for diabetic nephropathy. Diabetes Research and Clinical Practice, Limerick, v. 97, n. 3, p. 418-424, 2012.

Pourghasem, M.; Nasiri, E.; SHAFI, H. Early renal histological changes in alloxan-induced diabetic rats. International Journal of Molecular and Cellular Medicine, Babol, v. 3, n. 1, p. 1115, 2014

RASCH, R. Prevention of diabetic glomerulopathy in streptozotocin diabetic rats by insulin treatment. Diabetologia, Berlin, v. 16, n. 5, p. 319-324, 1979.

ROSSING, P.; HOUGAARD, P.; PARVING, H. H. Risk factors for development of incipient and overt diabetic nephropathy in type 1 diabetic patients: a 10-year prospective observational study. Diabetes Care, Alexandria, v. 25, n. 5, p. 859-864, 2002. 
STEFFES, M. W. et al. Diabetic nephropathy in the uninephrectomized dog: microscopic lesions after one year. Kidney International, New York, v. 21, n. 5, p. 721-724, 1982.

STOLAR, M. Glycemic control and complications in type 2 diabetes mellitus. The American Journal of Medicine, Alexandria, v. 123, p. S3-S11, 2010. Supplement 3.

STRUBLE, A. L. et al. Systemic hypertension and proteinuria in dogs with diabetes mellitus. Journal of American Veterinary Medical Association, Schaumburg, v. 213, n. 6, p. 822-825, 1998.

SUSZTAK, K. et al. Glucose-induced reactive oxygen species cause apoptosis of podocytes and podocyte depletion at the onset of diabetic nephropathy. Diabetes, Alexandria, v. 55, n. 1, p. $225-$ 233, 2006.

VORA, J. P.; ANDERSON, S.; BRENNER, B. M. Pathogenesis of diabetic glomerulopathy: the role of glomerular hemodynamic factors. In: MOGENSEN, C. F. The kidney and hypertension in diabetes mellitus. 2. ed. Boston: Kluwer Academic, 1994. p. 223232.

WARRAM, J. H. et al. Effect of duration of type I diabetes on the prevalence of stages of diabetic nephropathy defined by urinary albumin/creatinine ratio. Journal of the American Society of Nephrology, Baltimore, v. 7, n. 7, p. 930-937, 1996.

ZATZ, R. et al. Predominance of hemodynamic rather than metabolic factors in the pathogenesis of diabetic glomerulopathy. In: Proceedings of the National Academy of Sciences of the United States of America, Washington, DC, v. 82, n. 17, p. 59635967, 1985.

ZATZ, R. et al. Prevention of diabetic glomerulopathy by pharmacological amelioration of glomerular capillary hypertension. Journal of Clinical Investigation, New Haven, v. 77, n. 6, p. 19251930, 1986. 